\title{
Robustness of quantum correlations against decoherence
}

\author{
Ming-Liang $\mathrm{Hu}^{1}$ and Heng Fan ${ }^{2}$ \\ ${ }^{1}$ School of Science, Xi'an University of Posts and Telecommunications, Xi'an 710061, China \\ ${ }^{2}$ Beijing National Laboratory for Condensed Matter Physics, \\ Institute of Physics, Chinese Academy of Sciences, Beijing 100190, China
}

\begin{abstract}
We study dynamics of nonclassical correlations by exactly solving a model consisting of two atomic qubits with spontaneous emission. We find that the nonclassical correlations defined by different measures give different qualitative characterizations of those correlations. The relative behaviors of those correlation measures are presented explicitly for various quantum states in the two-qubit atomic system. In particular, we find that the robustness of quantum correlations can be greatly enhanced by performing appropriate local unitary operations on the initial state of the system.
\end{abstract}

PACS numbers: 03.65.Ta, 03.67.-a, 03.65.Yz

Key Words: Entanglement; Measurement-induced disturbance; Quantum discord; Geometric measure of quantum discord

\section{INTRODUCTION}

Entanglement plays a key role and is a resource in quantum information processing (QIP). In the last decades, much efforts have been put into study it in various physical systems, see for example Refs. [1 [5]. While being widely considered to be something unique without classical counterpart, it is recently realized that entanglement reflects only one aspect of quantum correlation of a quantum state, there exist some other nonclassical correlations [6 10]. One example is a model of QIP in Ref. [11], which outperforms its classical counterpart while without any entanglement. It is proposed that quantum discord (QD) [ [] ], a different measure of quantum correlation, might be responsible for the power of this model [12]. Thus, entanglement and QD, and additionally some other measures all are important in describing nonclassical correlations for a quantum system.

It is well acceptable that decoherence will cause decreasing of entanglement which may induce failure of the algorithms and various protocols of QIP. On the other hand, it is also of fundamental interest to study the behavior of nonclassical correlations with decoherence. Recently, there are some studies in evaluating the relationships between nonclassical correlations quantified by QD and entanglement quantified by concurrence or entanglement of formation (EoF) under the action of decoherence [13 24]. Also the role of QD in identifying critical points of quantum phase transitions are studied 25 28]. For practical use, one may hope that the nonclassical correlations which are crucial for QIP can be maintained for sufficiently long time so that the designed tasks can be fulfilled. However, the unavoidable interaction of a realistic system with its surroundings always makes it decaying with time. Particularly, under certain circumstances, the entanglement of a bipartite state can even terminate abruptly in a finite time, a phenomenon termed as entanglement sudden death (ESD) by $\mathrm{Yu}$ and Eberly [4]. For nonclaccical correlations as measured by QD and other similar quantities, however, the sudden death phenomenon has not been observed (this may be good for QIP independent of entanglement), but their values may decay with time and become zero at discrete instants of time [14, 15]. Thus it is of great importance to find ways to preserve them when the system is in contact with an environment which leads to decoherence.

In this paper, we offer a comparative study of the relationships between entanglement and different measures of nonclassical correlations for a certain family of twoqubit states. The system we considered consists of two atomic qubits with spontaneous emission [29]. We will show that even in cases where entanglement disappears, nonclassical correlations persist in the whole time region. In this sense, they are more robust against decoherence than entanglement, implying that quantum algorithms based only on them may be more robust than those based on entanglement. Moreover, we will show by explicit examples that robustness of quantum correlations can be greatly enhanced by performing local unitary operations to the initial states, though it is well-known that those measures of correlations themselves are invariant under local unitary operations [6-8].

This paper is organized as follows. In Section II we recall some measures of correlations for a bipartite state. We will adopt the EoF as the measure of entanglement, and other nonclassical correlations will be quantified by the measurement induced disturbance (MID), QD and the geometric measure of QD (GMQD). In Section III, we introduce the model and compare characteristics of various correlation measures for a class of two-qubit states. We will also evaluate robustness of quantum correlations for different local unitary equivalent states. Finally, Section IV is devoted to a summary.

\section{MEASURES OF QUANTUM CORRELATIONS}

The basic and crucial problem for studying dynamics of correlations of a composite system is to characterize and quantify correlation from different point of views in that system. The motivation of this study is to find whether 
those physical systems can be a platform for various tasks of QIP. In this section, we will first list several measures of quantum correlations for the bipartite states, and those measures will be applied in our study of the two-qubit atomic system.

First, we still consider entanglement as one important quantum correlation. In order to study the entanglement dynamics for any two-qubit states, we adopt EoF [1] which is a well-accepted measure of entanglement. The definition is as follows,

$$
E=H\left(\frac{1+\sqrt{1-C^{2}}}{2}\right),
$$

where $H(\tau)=-\tau \log _{2} \tau-(1-\tau) \log _{2}(1-\tau)$ is the binary Shannon entropy, and $C$ is the time-dependent concurrence [2], which takes the form $C=\max \left\{0, \lambda_{1}-\right.$ $\left.\lambda_{2}-\lambda_{3}-\lambda_{4}\right\}$, where $\lambda_{i}(i=1,2,3,4)$ are the square roots of the eigenvalues of the spin-flipped operator $R=$ $\rho\left(\sigma^{2} \otimes \sigma^{2}\right) \rho^{*}\left(\sigma^{2} \otimes \sigma^{2}\right)$ arranged in decreasing order, with $\rho^{*}$ being the complex conjugation of $\rho$ in the standard basis, and $\sigma^{2}$ is one Pauli matrix.

Besides entanglement, let us consider some other quantum correlations. The so-called MID, proposed recently by Luo in Ref. [7], is defined as the difference of two quantum mutual information respectively of a given state $\rho$ shared by two parties $a$ and $b$ and the corresponding post-measurement state $\Pi(\rho)$,

$$
\operatorname{MID}(\rho)=I(\rho)-I(\Pi(\rho)),
$$

where the mutual information, $I(\rho)=S\left(\rho^{a}\right)+S\left(\rho^{b}\right)-$ $S(\rho)$, measures the total correlation, including both classical and quantum, for a bipartite state $\rho$. Here $S(\rho)=$ $-\operatorname{tr}\left(\rho \log _{2} \rho\right)$ denotes the von Neumann entropy, with $\rho^{a}$ and $\rho^{b}$ being the reduced density matrix of $\rho$ by tracing out $b$ and $a$, respectively. While quantum mutual information of the state $\Pi(\rho), I(\Pi(\rho))$, quantifies the classical correlation in $\rho$, with $\Pi(\rho)=\Sigma_{i j}\left(\Pi_{i}^{a} \otimes \Pi_{j}^{b}\right) \rho\left(\Pi_{i}^{a} \otimes \Pi_{j}^{b}\right)$, where the measurement is induced by the spectral resolutions of the reduced states $\rho^{a}=\Sigma_{i} p_{i}^{a} \Pi_{i}^{a}$ and $\rho^{b}=$ $\Sigma_{j} p_{j}^{b} \Pi_{j}^{b}$. In fact, $\Pi(\rho)$ is a classical state (a state is referred to as classical if it is invariant under local von Neumann measurement) for any complete set of projective measurements $\left\{\Pi_{i}^{a}\right\}$ and $\left\{\Pi_{j}^{b}\right\}$. $\Pi(\rho)$ defined above is actually the closest classical state to $\rho$ since this kind of measurement leaves the marginal information invariant and is in a certain sense the least disturbing [7].

Another well-accepted measure of nonclassical correlation is the QD [6]. It was defined by the discrepancy between quantum mutual information and the classical aspect of correlation which was defined as the maximum information about one subsystem that can be obtained by performing a measurement on the other subsystem. If we restrict ourselves to the projective measurements performed locally on subsystem $a$ described by a complete set of orthogonal projectors $\left\{\Pi_{k}\right\}$, then the quantum state after measurement changes to $\rho_{b \mid k}=$ $\left(\Pi_{k} \otimes \mathbb{I}\right) \rho\left(\Pi_{k} \otimes \mathbb{I}\right) / p_{k}$, where $\mathbb{I}$ is the identity operator for subsystem $b$, and $p_{k}=\operatorname{tr}\left[\left(\Pi_{k} \otimes \mathbb{I}\right) \rho\left(\Pi_{k} \otimes \mathbb{I}\right)\right]$ is the probability for obtaining the measurement outcome $k$ on $a$. The classical correlation can then be obtained by maximizing $J\left(\rho \mid\left\{\Pi_{k}\right\}\right)=S\left(\rho^{b}\right)-S\left(\rho \mid\left\{\Pi_{k}\right\}\right)$ over all $\left\{\Pi_{k}\right\}$, where $S\left(\rho \mid\left\{\Pi_{k}\right\}\right)=\Sigma_{k} p_{k} S\left(\rho_{b \mid k}\right)$ is a generalization of the classical conditional entropy of subsystem $b$. Explicitly, the QD is defined as the minimal difference between $I(\rho)$ and $J\left(\rho \mid\left\{\Pi_{k}\right\}\right)$ as

$$
\mathrm{QD}(\rho)=I(\rho)-\sup _{\left\{\Pi_{k}\right\}} J\left(\rho \mid\left\{\Pi_{k}\right\}\right),
$$

where the supremum is taken over the complete set of $\left\{\Pi_{k}\right\}$. The intuitive meaning of QD thus may be interpreted as the minimal loss of correlations due to measurement. It vanishes for states with only classical correlation and survives for states with quantum correlation.

Since analytical expressions of QD are achievable only for certain special classes of states [21, 22], Dakić et al. [8] proposed the GMQD. They use the square of the Hilbert-Schmidt norm as the distance between two quantum states, and the nearest distance between $\rho$ and all of the zero-discord states $\Omega_{0}$ is interpreted as a measure of quantum correlation, which is defined as

$$
\operatorname{GMQD}(\rho)=\min _{\chi \in \Omega_{0}}\|\rho-\chi\|^{2}
$$

where the geometric quantity $\|\rho-\chi\|^{2}=\operatorname{tr}(\rho-\chi)^{2}$.

By noting that any two-quit state $\rho$ can be represented as $\rho=\frac{1}{4}\left(\mathbb{I} \otimes \mathbb{I}+\vec{x} \cdot \vec{\sigma} \otimes \mathbb{I}+\mathbb{I} \otimes \vec{y} \cdot \vec{\sigma}+\sum_{i, j=1}^{3} r_{i j} \sigma_{i} \otimes \sigma_{j}\right)$, Dakić et al. derived an explicit formula of GMQD, which is given by [8]

$$
\operatorname{GMQD}(\rho)=\frac{1}{4}\left(\|x\|^{2}+\|R\|^{2}-k_{\text {max }}\right),
$$

where the scalar product $\vec{\alpha} \cdot \vec{\sigma}=\sum_{i=1}^{3} \alpha_{i} \sigma_{i}$ with $\alpha=x, y$. Moreover, $\|x\|^{2}=\sum_{i=1}^{3} x_{i}^{2},\|R\|^{2}=\operatorname{tr}\left(R^{T} R\right)$, and $k_{\max }$ is the largest eigenvalue of the matrix $K=x x^{T}+R R^{T}$, where the superscript $T$ denotes transpose of vectors or matrices. Since this measure gives analytic results without restriction on the form of $\rho$, it turns out to be a convenient tool for analyzing quantum correlation dynamics from a geometric perspective.

Correlation measures (i.e.,EoF, MID and QD) listed above assume equal values for bipartite pure states, but this is not the case for mixed states. Particularly, the QD and GMQD are not symmetric quantities with respect to the measurements performed on subsystem $a$ or $b$, and the state is said to be completely classically correlated only when both of them reach zero. In the subsequent discussions, however, we will restrict ourselves to the situation that the states are symmetric under exchange of subsystems. 


\section{CORRELATION DYNAMICS UNDER DECOHERENCE}

We are interested in revealing difference between various correlation measures under the action of noisy environments. For this purpose, we consider a system consists of two identical atoms with spontaneous emission, and having lower and upper levels $\left|g_{i}\right\rangle$ and $\left|e_{i}\right\rangle(i=1,2)$ separated by the energy gap $\hbar \omega$, with $\omega$ being the transition frequency. Also, we assume this system is coupled to a multimode vacuum electromagnetic field, under the influence of which its time evolution is governed by the following master equation [29, 30]

$$
\begin{aligned}
\frac{\partial \rho}{\partial t}= & -\mathrm{i} \omega \sum_{i=1}^{2}\left[S_{i}^{z}, \rho\right]-\mathrm{i} \sum_{i \neq j}^{2} \Omega_{i j}\left[S_{i}^{+} S_{j}^{-}, \rho\right] \\
& +\frac{1}{2} \sum_{i, j=1}^{2} \gamma_{i j}\left(2 S_{j}^{-} \rho S_{i}^{+}-\left\{S_{i}^{+} S_{j}^{-}, \rho\right\}\right),
\end{aligned}
$$

where $S_{i}^{ \pm}$are the raising and lowering operators while $S_{i}^{z}$ is the energy operator. $\gamma_{i j} \equiv \gamma(i=j)$ are the spontaneous emission rates of the atoms caused by their direct interaction with the vacuum field. Moreover, $\gamma_{i j}$ and $\Omega_{i j}$ $(i \neq j)$ describe the collective damping and the dipoledipole interaction potential, respectively. They both depend on the interatomic distance $r_{i j}=\left|r_{j}-r_{i}\right|$ and take the form

$$
\begin{aligned}
\gamma_{i j} & =\frac{3}{2} \gamma\left[\frac{\sin \left(k r_{i j}\right)}{k r_{i j}}+\frac{\cos \left(k r_{i j}\right)}{\left(k r_{i j}\right)^{2}}-\frac{\sin \left(k r_{i j}\right)}{\left(k r_{i j}\right)^{3}}\right], \\
\Omega_{i j} & =\frac{3}{4} \gamma\left[\frac{\sin \left(k r_{i j}\right)}{\left(k r_{i j}\right)^{2}}+\frac{\cos \left(k r_{i j}\right)}{\left(k r_{i j}\right)^{3}}-\frac{\cos \left(k r_{i j}\right)}{k r_{i j}}\right],
\end{aligned}
$$

where we have assumed that the atomic dipole moments for the two atoms take the same value (i.e., $\mu_{1}=\mu_{2}=\mu$ ) and are polarized in the direction perpendicular to the interatomic axis. And, $k=2 \pi / \lambda$ is the wave vector with $\lambda$ being the atomic resonant wavelength.

We first consider the Bell-like initial state as follows

$$
|\Psi\rangle=\alpha\left|e_{1} e_{2}\right\rangle+\sqrt{1-\alpha^{2}}\left|g_{1} g_{2}\right\rangle,
$$

for which the nonzero elements of $\rho(t)$ in the standard basis $\left\{\left|e_{1} e_{2}\right\rangle,\left|e_{1} g_{2}\right\rangle,\left|g_{1} e_{2}\right\rangle,\left|g_{1} g_{2}\right\rangle\right\}$ are of the form

$$
\begin{aligned}
\rho_{11}(t) & =\alpha^{2} e^{-2 \gamma t}, \\
\rho_{14}(t) & =\rho_{41}^{*}(t)=\alpha \sqrt{1-\alpha^{2}} e^{-(\gamma+2 \mathrm{i} \omega) t}, \\
\rho_{22,33}(t) & =a_{1}\left[e^{-\gamma_{12}^{+} t}-e^{-2 \gamma t}\right]+a_{2}\left[e^{-\gamma_{12}^{-} t}-e^{-2 \gamma t}\right], \\
\rho_{23,32}(t) & =a_{1}\left[e^{-\gamma_{12}^{+} t}-e^{-2 \gamma t}\right]-a_{2}\left[e^{-\gamma_{12}^{-} t}-e^{-2 \gamma t}\right], \\
\rho_{44}(t) & =1-\rho_{11}(t)-\rho_{22}(t)-\rho_{33}(t),
\end{aligned}
$$

where $\gamma_{12}^{ \pm}=\gamma \pm \gamma_{12}, a_{1,2}=\alpha^{2} \gamma_{12}^{ \pm} / 2 \gamma_{12}^{\mp}$. As one can see, the X structure of $\rho(t)$ is maintained during the evolution, which greatly facilitates the following analysis.

Fig. 1 shows the contour plots of the quantum correlations versus $\gamma t$ and $\alpha^{2}$ in the short time region with the
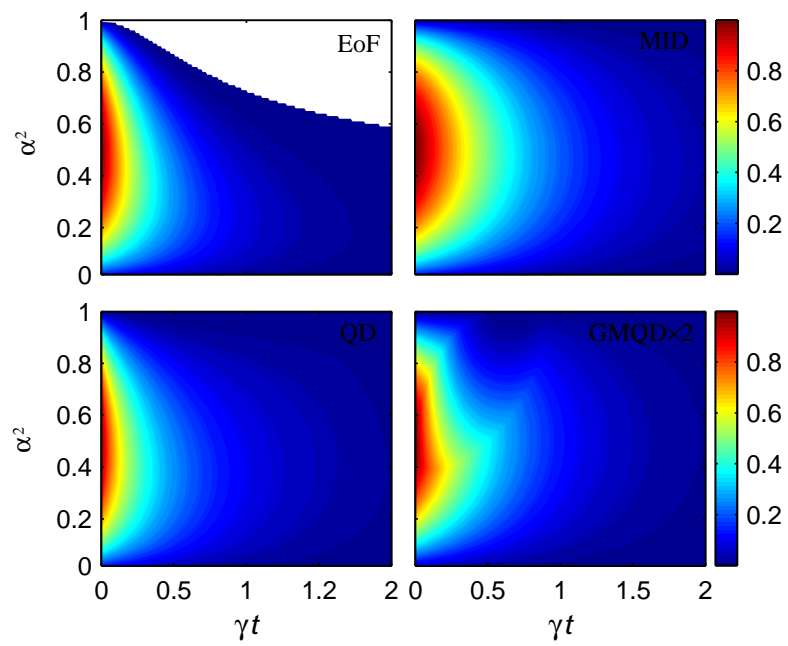

FIG. 1: (Color online) Contour plots of different correlation measures versus $\gamma t$ and $\alpha^{2}$ for the initial state $|\Psi\rangle$, where the interatomic distance for these plots is $r_{12}=0.6737 \lambda$.

interatomic distance $r_{12}=0.6737 \lambda$, at which $\gamma_{12}$ reaches its minimal value. Clearly, while the ESD happens after a finite time, the MID, QD and GMQD maintain during the whole time region. This points to a fact that the nonclassical correlations may be more resistant to external perturbations than that of entanglement. Moreover, the GMQD shows a revival for large values of $\alpha^{2}$, but during the same time region the other correlation measures are always decreased. Thus similar to the relativity of different entanglement measures (see [31] and references therein), the nonclassical correlation measures may also impose different orderings of quantum states.

For quantum states of Eq. (9), the concurrence can be obtained analytically as $C(\rho)=2 \max \left\{0, C_{1}, C_{2}\right\}$, where $C_{1}=\left|\rho_{14}\right|-\sqrt{\rho_{22} \rho_{33}}$ and $C_{2}=\left|\rho_{23}\right|-\sqrt{\rho_{11} \rho_{44}}$. In the short time region, $\left|\rho_{14}\right|^{2}>\rho_{22} \rho_{33}$, thus the concurrence as well as EoF are continuous functions of $\gamma t$. In the long time region, $\left|\rho_{23}\right|^{2}>\rho_{11} \rho_{44}$, and the entanglement may experiences a very weak revival after a finite time interval of its complete disappearance. Similarly, for $\rho(t)$ of Eq. (9) we have $\operatorname{MID}(\rho)=S(\Pi(\rho))-S(\rho)$, with $\Pi(\rho)=\operatorname{diag}\left\{\rho_{11}, \rho_{22}, \rho_{33}, \rho_{44}\right\}$, thus it is understandable that the MID also behaves as continuous functions of $\gamma t$ and $\alpha^{2}$. For the QD and GMQD, as denoted by the blue and green curves in Fig. 2, they present sudden changes at discrete instants of time or $\alpha^{2}$ which are evidenced by the presence of kinks. In general, these sudden change behaviors are caused by the optimal procedure for choosing the measurement operators, and we will discuss this issue in detail in the following.

From Fig. 2 one can also observe that the MID, QD and GMQD behave as non-monotonic decreasing functions of $\gamma t$ and $\alpha^{2}$. Their values may be increased or decreased during certain parameter regions. Particularly, it is worthwhile to note that there are states having different orderings induced by different correlation measures. 

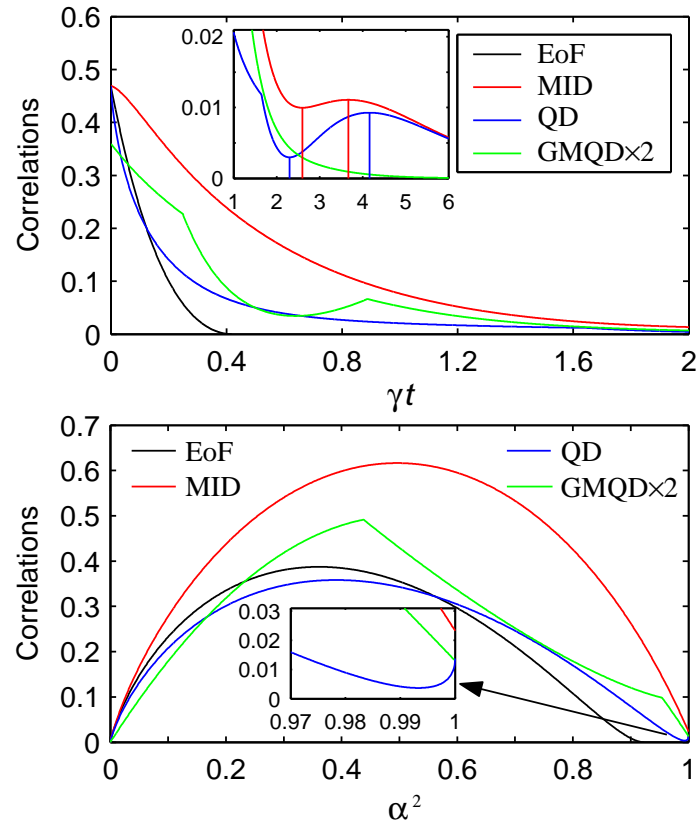

FIG. 2: (Color online) Quantum correlations versus $\gamma t$ with $\alpha^{2}=0.9$ (top) and versus $\alpha^{2}$ with $\gamma t=0.35$ (bottom) for the initial state $|\Psi\rangle$ with $r_{12}=0.6737 \lambda$. The insets show the cases when $\gamma t \in[1,6]$ and $\alpha^{2} \in[0.97,1]$, where the curves are cut to better visualize of the revival phenomenon, and the vertical lines indicate the critical $\gamma t$ at which the correlations turn out to be increased or decreased.

For example, for the parameters chosen in Fig. 2 there exists states $\rho_{1}$ and $\rho_{2}$ such that $\operatorname{MID}\left(\rho_{1}\right)>\operatorname{MID}\left(\rho_{2}\right)$, $\mathrm{QD}\left(\rho_{1}\right)>\mathrm{QD}\left(\rho_{2}\right)$, while $\operatorname{GMQD}\left(\rho_{1}\right)<\operatorname{GMQD}\left(\rho_{2}\right)$ when $\gamma t \in[0.639,0.888]$, i.e., the decrease of MID and QD are accompanied by the increase of GMQD. Moreover, from the insets of Fig. 2, other states differently ordered by the nonclassical correlation measures are readily distinguished, including those during the scaled time regions $\gamma t \in[2.304,2.604]$ and $\gamma t \in[3.672,4.164]$, for which we have $\operatorname{MID}\left(\rho_{1}\right)>\operatorname{MID}\left(\rho_{2}\right)$ and $\operatorname{GMQD}\left(\rho_{1}\right)>$ $\operatorname{GMQD}\left(\rho_{2}\right)$, while $\mathrm{QD}\left(\rho_{1}\right)<\mathrm{QD}\left(\rho_{2}\right)$. Thus we see that there are no simple dominance relations between MID, $\mathrm{QD}$ and GMQD for general cases. They are different not only quantitatively but also qualitatively.

Now we evaluate analytically the sudden change behaviors of the QD. For $\rho(t)$ in Eq. (9), the infimum of the conditional entropy can be determined as

$$
\inf _{\{\theta, \phi\}} S\left(\rho \mid\left\{\Pi_{k}\right\}\right)=H(\tau),
$$

where the optimal angle $\theta$ related to the projective measurement is $\theta=(m+0.5) \pi$ with $m \in \mathbb{Z}$, and $H(\tau)$ is the Shannon entropy functional, with

$$
\tau=\frac{1-\sqrt{\left[1-2\left(\rho_{11}+\rho_{33}\right)\right]^{2}+4\left(\left|\rho_{14}\right|^{2}+\left|\rho_{23}\right|^{2}+\delta\right)}}{2},
$$

with $\delta=2 \alpha\left(1-\alpha^{2}\right)^{1 / 2} e^{-\gamma t} \rho_{23} \cos (2 \phi-2 \omega t)$. Clearly, the infimum of $S\left(\rho \mid\left\{\Pi_{k}\right\}\right)$ is obtained whenever $\phi=k \pi+\omega t$
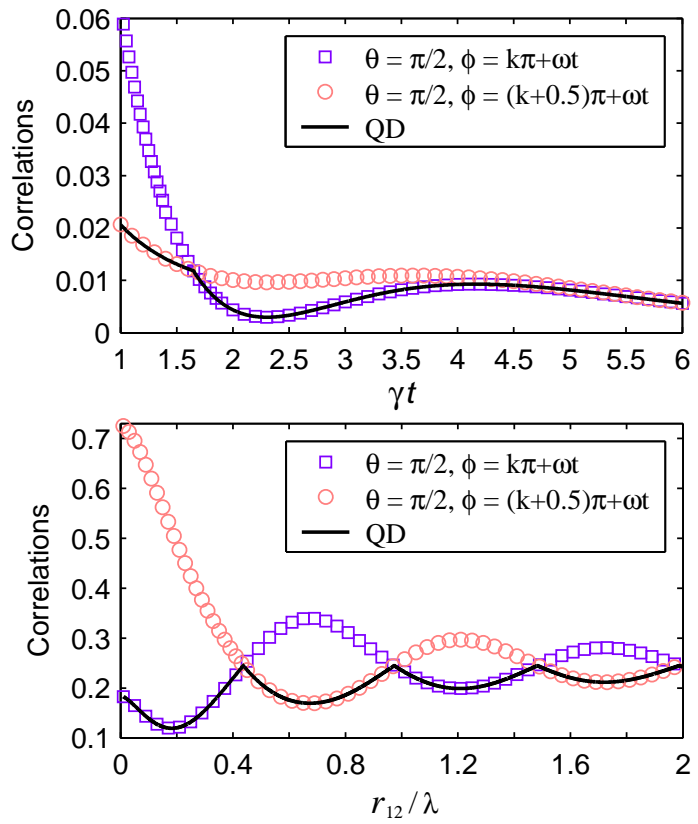

FIG. 3: (Color online) QD versus $\gamma t$ with $r_{12}=0.6737 \lambda$, $\alpha^{2}=0.9$ (top) and versus $r_{12} / \lambda$ with $\gamma t=0.35, \alpha^{2}=0.8$ (bottom) for the initial state $|\Psi\rangle$.

if $\alpha \rho_{23}>0$, and $\phi=(k+0.5) \pi+\omega t(k \in \mathbb{Z})$ if $\alpha \rho_{23}<0$. Thus we see that the sudden change behaviors of QD here is caused by the optimization procedure for choosing the optimal measurement angle $\phi$ or equivalently, the optimal projective measurement operators $\left\{\Pi_{k}\right\}$ because for the two-qubit case $\Pi_{k}$ can be expressed as $\Pi_{1}=(\mathbb{I}+\vec{n} \cdot \vec{\sigma}) / 2$ and $\Pi_{2}=\mathbb{I}-\Pi_{1}$, with $\vec{n}=(\sin \theta \cos \phi, \sin \theta \sin \phi, \cos \theta)^{T}$ being a unit vector in $\mathbb{R}^{3}$. Here it should be emphasized that the angle $\phi$ that minimizes the QD is time dependent, which is different from the previous results with real elements of the density matrix [13 17]. As is shown evidently in the top panel of Fig. 3 with fixed $r_{12}$ and $\alpha^{2}$, the optimal measurement angle $\phi$ assumes the value $\phi=(k+0.5) \pi+\omega t$ in the short time region, while in the long time region it is $\phi=k \pi+\omega t$. For fixed $\gamma t$ and $\alpha^{2}$, as exposed in the bottom panel of Fig. 3, the two different choices of $\phi$ yields the QD alternatively.

When considering the GMQD, from Eq. (5) it is readily to see that it can also be expressed as $\operatorname{GMQD}(\rho)=$ $\left(\operatorname{tr} K-k_{\max }\right) / 4$, where $\operatorname{tr} K=\Sigma_{i} k_{i}$ and $k_{\max }=$ $\max \left\{k_{1}, k_{2}, k_{3}\right\}$. For quantum states with the $\mathrm{X}$ structure (of course, including those of the form of Eq. (9)) one can obtain the eigenvalues of $K$ analytically as

$$
\begin{aligned}
k_{1,2} & =4\left(\left|\rho_{14}\right| \pm\left|\rho_{23}\right|\right)^{2}, \\
k_{3} & =2 \sum_{n=1}^{4} \rho_{n n}^{2}-4\left(\rho_{11} \rho_{33}+\rho_{22} \rho_{44}\right) .
\end{aligned}
$$

The $k_{i}$ are in arbitrary order and cannot be ordered by magnitude unless the parameter values are known, thus $k_{\max }$ may be changed with the variation of the parameters involved, which causes the discontinuities of the 

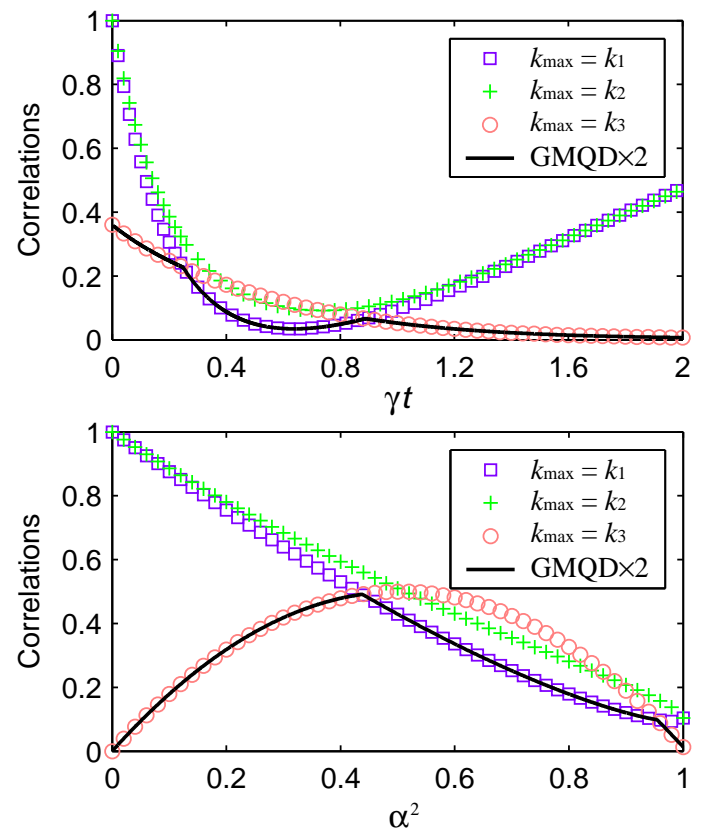

FIG. 4: (Color online) GMQD versus $\gamma t$ with $\alpha^{2}=0.9$ (top) and versus $\alpha^{2}$ with $\gamma t=0.35$ (bottom) for the initial state $|\Psi\rangle$ with the interatomic distance $r_{12}=0.6737 \lambda$.

GMQD. Two exemplified plots for certain specified system parameters are presented in Fig. 4, from which one can see that for fixed $\alpha^{2}=0.9$, the GMQD is obtained when $k_{\max }=k_{3}$ in the short and long time regions, while in the middle time region, it is obtained when $k_{\max }=k_{1}$. For fixed $\gamma t=0.35$, we have $k_{\max }=k_{3}$ for small or large values of $\alpha^{2}$, and $k_{\max }=k_{1}$ for middle values of $\alpha^{2}$.

In Ref. [8] Dakić et al. showed that $k_{\max }$ can be written as $k_{\max }=\max _{|\vec{e}=1|} \vec{e}^{T} K \vec{e}$, and the sudden change in decay rates of GMQD corresponds to the sudden change of the optimized $\vec{e}=(\sin \theta \cos \phi, \sin \theta \sin \phi, \cos \theta)^{T}$ in $\mathbb{R}^{3}$. For the two-qubit case, the closest zero-discord state $\chi=\Sigma_{k=1}^{2} p_{k} \Pi_{k} \otimes \rho_{k}$ is obtained when $\Pi_{1}=(\mathbb{I}+\vec{e} \cdot \vec{\sigma}) / 2$ and $\Pi_{2}=\mathbb{I}-\Pi_{1}$, with $\vec{e}$ being the eigenvector of $K$ with the largest eigenvalue. The eigenvectors correspond to the three eigenvalues of Eq. (12) are

$$
\begin{aligned}
\left|\psi_{1,2}\right\rangle & =a_{1,2}\left(1, \frac{\operatorname{Re}\left(\rho_{14} \rho_{23}\right) \mp\left|\rho_{14} \rho_{23}\right|}{\operatorname{Im}\left(\rho_{14} \rho_{23}\right)}, 0\right)^{T}, \\
\left|\psi_{3}\right\rangle & =(0,0,1)^{T},
\end{aligned}
$$

where $a_{1,2}$ are normalization constants. $\vec{e}$ assumes $\left|\psi_{1}\right\rangle$ or $\left|\psi_{3}\right\rangle$ with the variation of the involved system parameters. If $\vec{e}=\left|\psi_{1}\right\rangle$ then we have $\cos \theta=0$ or equivalently, $\theta=(n+0.5) \pi$ with $n \in \mathbb{Z}$. If $\vec{e}=\left|\psi_{3}\right\rangle$, however, we have $\cos \theta= \pm 1$ or $\theta=n \pi$ with $n \in \mathbb{Z}$. The optimal $\phi$ can also be derived exactly, and here we do not care about it for the sudden change behaviors of GMQD have already been reflected by the discontinuity of the optimal $\theta$.

Notice that although both the optimal unit vectors $\vec{n}$ for the QD and $\vec{e}$ for the GMQD are related to the projective operators, they are not the same for general case,

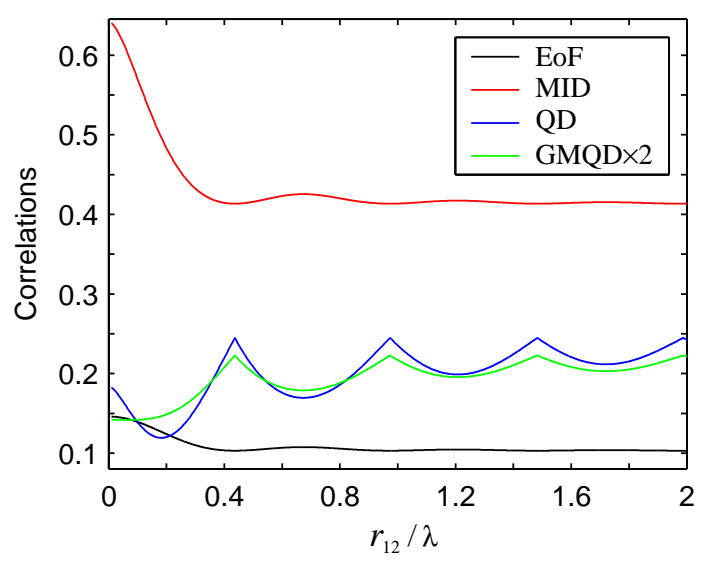

FIG. 5: (Color online) Quantum correlations versus $r_{12} / \lambda$ for the initial state $|\Psi\rangle$ with $\alpha^{2}=0.8$ and $\gamma t=0.35$.
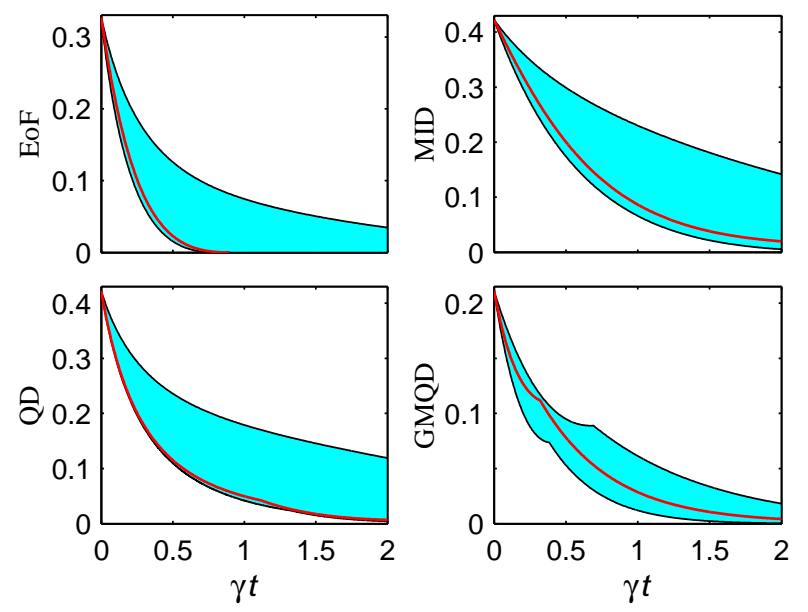

FIG. 6: (Color online) Quantum correlations versus $\gamma t$ for the initial state $\rho^{\Psi}(0)=p|\Psi\rangle\langle\Psi|+(1-p) \mathbb{I} / 4$ with $\alpha^{2}=1 / 2$, $p=0.65$ and $r_{12}=0.6737 \lambda$ (red curves). The cyan regions show the extent to which the correlations can be adjusted by performing operations $\{\mathbb{I} \times V\}$ to the second qubit.

which is reflected in the disagreement of their individual sudden changes in the decay rates.

In Fig. 5 we show the influence of $r_{12} / \lambda$ on correlation dynamics for the initial state $|\Psi\rangle$ with $\alpha^{2}=0.8$ and $\gamma t=0.35$. It is seen that all the correlation measures behave as damped oscillations with increasing $r_{12} / \lambda$, and the dependence of QD and GMQD on $r_{12} / \lambda$ are more sensitive than those of EoF and MID. Also one can note that there exists states $\rho_{1}$ and $\rho_{2}$ such that $\mathrm{QD}\left(\rho_{1}\right)>\mathrm{QD}\left(\rho_{2}\right)$, $\operatorname{GMQD}\left(\rho_{1}\right)>\operatorname{GMQD}\left(\rho_{2}\right)$ and $\operatorname{MID}\left(\rho_{1}\right)<\operatorname{MID}\left(\rho_{2}\right)$. Thus we see again that the states have different orderings induced by different measures of nonclassical correlations.

Finally, we show that the robustness of quantum correlations may be enhanced by performing local unitary operations to the initial state $\rho(0)$. As we know, the correlation measures (EoF, MID, QD and GMQD) are locally 
unitary invariant; that is, $\rho(0)$ and $(U \otimes V) \rho(0)\left(U^{\dagger} \otimes V^{\dagger}\right)$ have completely the same values of correlations for any unitary operators $U$ and $V$ acting on parties $a$ and $b$. But this does not mean that their robustness against decoherence are the same. In fact, for any initial state $\rho(0)$, there exist an optimal local unitary transformation $U_{\text {opt }} \otimes V_{\text {opt }}$ which can enhance the robustness of the correlations to a certain greatest extent. However, in general, the optimal $U_{\text {opt }} \otimes V_{\text {opt }}$ is determined by the decoherence mechanism as well as the explicit form of $\rho(0)$, and it is difficult to obtain an analytical expression of it.

For simplicity, we consider the initial state as a Wernerlike state $\rho^{\Psi}(0)=p|\Psi\rangle\langle\Psi|+(1-p) \mathbb{I} / 4$. And we restrict ourselves to the complete set of locally unitary transformations $\{\mathbb{I} \times V\}$ (a subset of $\{U \otimes V\}$ ). Density matrix $\rho^{\Psi}(t)$ for $\rho^{\Psi}(0)$ can be obtained analytically [30], and the dependence of EoF, MID, QD as well as GMQD on the elements of $\rho^{\Psi}(t)$ are the same as those for the initial state $|\Psi\rangle$. But the parameter appeared in Eq. (11) changes to $\delta=2\left|\rho_{14}\right|\left|\rho_{23}\right| \cos \left(2 \phi-\omega_{1}-\omega_{2}\right)$, where $\cos \omega_{1,2}=$ $\operatorname{Re}\left(\rho_{14,23}\right) /\left|\rho_{14,23}\right|$ and $\sin \omega_{1,2}=-\operatorname{Im}\left(\rho_{14,23}\right) /\left|\rho_{14,23}\right|$. For general $\rho(0) \in\left\{(\mathbb{I} \times V) \rho^{\Psi}(0)\left(\mathbb{I} \times V^{\dagger}\right)\right\}$, however, the density matrix $\rho(t)$ as well as the correlation measures can only be obtained numerically since the $\mathrm{X}$ structure of it may be destroyed under the transformation $\{\mathbb{I} \times V\}$. By expressing $V$ in terms of the Pauli operators as $V=\sin \alpha\left(\sigma^{1} \cos \gamma+\sigma^{2} \sin \gamma\right)+\cos \alpha\left(\sigma^{3} \cos \beta-\mathrm{i} \sigma^{0} \sin \beta\right)$ with $\alpha \in[0, \pi / 2]$ and $\beta, \gamma \in[0,2 \pi]$, we perform numerical calculations with $p=0.65, \alpha^{2}=1 / 2, r_{12}=0.6737 \lambda$ and show the results in Fig. 6. It is shown that the extent of different correlation measures at finite time $t>0$ can be adjusted among values bounded by the cyan regions by performing operations $\{\mathbb{I} \times V\}$ to the initial state. Particularly, the sudden death phenomenon of entanglement may be avoided. Thus we see that different locally unitary equivalent states show different robustness against decoherence induced by the spontaneous emission, and this provides a way for long-time preservation of quantum correlations in this system.

\section{CONCLUSIONS}

In conclusion, we evaluated systematically the quantum correlations quantified by the EoF, MID, QD and
GMQD by exactly solving a model with the two qubits interacting with a multimode radiation field. We showed that the dynamics for these quantities may be rather different. Particularly, while entanglement experiences sudden death, MID, QD and GMQD persist for an infinite time, i.e., they are more robust than that of entanglement against decoherence, which is important for QIP tasks which deos not based on entanglement. Moreover, the dissipative process of spontaneous emission may lead to a generation of states manifesting the relativity of different correlation measures. In general, different measures of nonclassical correlations are incomparable since their behaviors may be qualitatively different.

We also demonstrated the disagreement between $\mathrm{QD}$ and GMQD on reflecting the sudden change behaviors of nonclassical correlations of a given quantum state. The origin of this phenomenon is the different definitions of discord based on different measures of distance between two states. While the QD is based on the von Neumann entropy, the GMQD is based on the Hilbert-Schmidt norm, and it is this difference that yields different optimal measurement operators.

Since it is generally accepted that quantum correlations is crucial for QIP, it is important to maintain them as long as possible. Here we showed that although local unitary operations cannot change extents of quantum correlations of a state, it can greatly enhance the robustness of a system against decoherence. This provides a possible way for long-time preservation of the quantum correlations by local unitary operations.

\section{ACKNOWLEDGMENTS}

This work was supported by NSFC (10974247), "973" program (2010CB922904), NSF of Shaanxi Province (2010JM1011, 2009JQ8006), and the Scientific Research Program of Education Department of Shaanxi Provincial Government (2010JK843).
[1] C.H. Bennett, D.P. DiVincenzo, J.A. Smolin, W.K. Wootters, Phys. Rev. A 54 (1996) 3824.

[2] W.K. Wootters, Phys. Rev. Lett. 80 (1998) 2245.

[3] R. Horodecki, P. Horodecki, M. Horodecki, K. Horodecki, Rev. Mod. Phys. 81 (2009) 865.

[4] T. Yu, J.H. Eberly, Phys. Rev. Lett. 93 (2004) 140404; T. Yu, J.H. Eberly, Phys. Rev. Lett. 97 (2006) 140403.

[5] L. Amico, R. Fazio, A. Osterloh, V. Vedral, Rev. Mod. Phys. 80 (2008) 517.

[6] H. Ollivier, W.H. Zurek, Phys. Rev. Lett. 88 (2001)
017901; L. Henderson, V. Vedral, J. Phys. A 34 (2001) 6899.

[7] S. Luo, Phys. Rev. A 77 (2008) 022301.

[8] B. Dakić, V. Vedral, Č. Brukner, Phys. Rev. Lett. 105 (2010) 190502.

[9] K. Modi, T. Paterek, W. Son, V. Vedral, M. Williamson, Phys. Rev. Lett. 104 (2010) 080501.

[10] A. Streltsov, H. Kampermann, D. Bruß, Phys. Rev. Lett. 106 (2011) 160401.

[11] E. Knill, R. Laflamme, Phys. Rev. Lett. 81 (1998) 5672. 
[12] A. Datta, A. Shaji, C.M. Caves, Phys. Rev. Lett. 100 (2008) 050502.

[13] T. Werlang, S. Souza, F.F. Fanchini, C.J. Villas Boas, Phys. Rev. A 80 (2009) 024103.

[14] B. Wang, Z.Y. Xu, Z.Q. Chen, M. Feng, Phys. Rev. A 81 (2010) 014101.

[15] F.F. Fanchini, T. Werlang, C.A. Brasil, L.G.E. Arruda, A.O. Caldeira, Phys. Rev. A 81 (2010) 052107.

[16] J. Maziero, L.C. Céleri, R.M. Serra, V. Vedral, Phys. Rev. A 80 (2009) 044102.

[17] X.M. Lu, Z.J. Xi, Z. Sun, X.G. Wang, Quantum Inf. Comput. 10 (2010) 0994.

[18] Y. Li, B. Luo, H. Guo, Phys. Rev. A 84 (2011) 012316.

[19] A. Ferraro, L. Aolita, D. Cavalcanti, F.M. Cucchietti, A. Acín, Phys. Rev. A 81 (2010) 052318.

[20] S. Luo, S. Fu, Phys. Rev. A 82 (2010) 034302.

[21] S. Luo, Phys. Rev. A 77 (2008) 042303.

[22] M. Ali, A.R.P. Rau, G. Alber, Phys. Rev. A 81 (2010)
042105; D. Girolami, G. Adesso, Phys. Rev. A 83 (2011) 052108.

[23] M.D. Lang, C.M. Caves, Phys. Rev. Lett. 105 (2010) 150501; B. Li, Z.X. Wang, S.M. Fei, Phys. Rev. A 83 (2011) 022321.

[24] L. Mazzola, J. Piilo, S. Maniscalco, Phys. Rev. Lett. 104 (2010) 200401.

[25] T. Werlang, C. Trippe, G.A.P. Ribeiro, G. Rigolin, Phys. Rev. Lett. 105 (2010) 095702.

[26] M.S. Sarandy, Phys. Rev. A 80 (2009) 022108.

[27] B.Q. Liu, B. Shao, J.G. Li, J. Zou, L.A. Wu, Phys. Rev. A 83 (2011) 052112.

[28] Y.C. Li, H.Q. Lin, Phys. Rev. A 83 (2011) 052323.

[29] Z. Ficek, S. Swain, Quantum Interference and Coherence: Theory and Experiments, Spring, New York, 2005.

[30] Z. Ficek, R. Tanaś, Phys. Rev. A 74 (2006) 024304.

[31] A. Miranowica, Phys. Lett. A 327 (2004) 272. 